\title{
Intrauterine growth restriction in pregnant women after kidney transplantation as a marker of preeclampsia
}

\author{
Anna Cyganek, Filip A. Dabrowski, Bronislawa Pietrzak, Zoulikha Jabiry-Zieniewicz, \\ Barbara Grzechocinska, Anna Madej, Miroslaw Wielgos \\ ${ }^{\text {st }}$ Department of Obstetrics and Gynecology, Medical University of Warsaw, Poland
}

\begin{abstract}
Objectives: Delayed motherhood is associated with an increasing number of comorbidities such as glomerulonephritis, systemic lupus erythematosus, and diabetic nephropathy. Women after renal transplant belong to the group of patients who require a highly individualized approach to treatment and diagnosis. The aim of the study was to validate the commonly used diagnostic criteria for preeclampsia which seem to be irrelevant in patients with chronic renal insufficiency.

Material and methods: The course of pregnancy and delivery were retrospectively analyzed in 48 renal transplant patients. Two patients were excluded. Group I included 23 patients with eutrophic neonates, while Group II consisted of 23 patients with fetal hypotrophy (birth weight of $<10^{\text {th }}$ percentile).

Results: The duration of pregnancy was 34.5 and 35 weeks in Groups I and II, respectively. Mean birth weight in Groups I and II was $2608.64 \mathrm{~g}$ and $2046.30 \mathrm{~g}$, respectively $(\mathrm{p}=0.002)$. Mean weight percentile in Groups I and II was 36.57 and 2.91 , respectively $(p<0.000)$. Proteinuria in the first half of pregnancy occurred in 16 and 14 patients from Groups I and II, respectively, and increased in the second half of pregnancy in 6 and 6 patients from Groups I and II, respectively. Patients from Group II were more prone to urinary tract infections ( 0.43 vs. $0.79 ; p=0.02$ ).

Conclusions: Current diagnostic criteria for preeclampsia are insufficient in case of pregnant women after kidney transplant. General criteria should be applied with special care in women with chronic kidney disease or in patients with systemic lupus erythematosus. As a predictive factor of neonatal morbidity, intrauterine growth restriction seems to be more valuable than typical markers of kidney function.
\end{abstract}

Key words: intrauterine growth restriction, kindey transplantation, preeclampsia, cyclosporine, tacrolimus

Ginekologia Polska 2016; 87, 11: 769-772

\section{INTRODUCTION}

Recent years have witnessed significant changes in the classification of hypertension and preeclampsia in pregnant women. The 2014 recommendations of the International Society for the Study of Hypertension in Pregnancy (ISSHP) and the 2013 guidelines of the American Society of Obstetricians and Gynecologists (ACOG, the American College of Obstetricians and Gynecologists) have defined preeclampsia as hypertension occurring $>20$ weeks of pregnancy, accompanied by one of the following symptoms: thrombocytopenia $<150000 / d L$, deterioration of the liver function (doubled values of ALT and AST), renal failure (creatinine $>1.1 \mathrm{mg} / \mathrm{dL}$ ), and neurological disorders or blurred vision. The difference between the two classifications is that according to the ISSHP guidelines, intrauterine growth restriction (IUGR) is an additional factor which allows to diagnose preeclampsia. Also, IUGR has been recognized a part of the diagnostic process for preeclampsia in the November 9, 2015 Regulation of the Minister of Health of the Republic of Poland on the management standards in pathological pregnancies [1].

None of the abovementioned classifications [2, 3] takes into account the specific characteristics of patients with chronic kidney disease. Due to delayed motherhood, women with glomerulonephritis, systemic lupus erythe- 
matosus and diabetic nephropathy are becoming increasingly common in the obstetric practice. Women after renal transplantation are among patients who require a highly individualized approach to both, treatment and diagnosis. Therefore, they were chosen to represent diversity in the diagnosis of preeclampsia in pregnant women with chronic kidney disease. IUGR is a complication of pregnancy whose occurrence may be affected by numerous factors, e.g. environmental conditions, such as smoking, anemia, hypertension, as well as chronic use of immunosuppressive drugs and the nature of the transplanted organ. It is impossible to identify one factor as the main cause of IUGR, but in patients after renal transplant at least two of them - hypertension and immunosuppressive therapy - are seen virtually in each case. Thus, our study was an attempt to validate the commonly used diagnostic criteria and support the hypothesis that intrauterine hypotrophy as a reflection of the materno-placental interaction should be an independent and important marker of preeclampsia in patients with chronic kidney insufficiency.

\section{MATERIAL AND METHODS}

We analyzed retrospectively the course of pregnancy and delivery in 48 renal transplant patients, who delivered at the First Department of Obstetrics and Gynecology between 2002 and 2015. Two patients were excluded from the study, because they had neither gestational nor pre-gestational hypertension. One of them delivered an eutrophic and the other a hypotrophic baby. The remaining 46 women with pre-gestational hypertension were divided into two groups: 23 patients who delivered eutrophic children (Group I) and 23 patients diagnosed with fetal hypotrophy defined as birth weight of $<10$ percentile for the gestational age (Group II). The following factors were analyzed: occurrence of preeclampsia, duration of pregnancy, mode of delivery and postnatal state of the neonate, and compared for the two groups.

\section{RESULTS}

No differences were found in terms of maternal age at delivery (30.8 vs. 29.17; $p=0.095)$, time from transplantation (5.41 vs. $4.6 ; p=0.163)$, BMI ( 27.35 vs. $26.29 ; p=0.17$ ) or immunosuppressive drug regimen $(p>0.05)$ between the groups. Mean duration of pregnancy was similar: 34.5 and 35 weeks in Groups I and II, respectively ( $p=0.235$ ). Mean birth weight was $2608.64 \mathrm{~g}$, and $2046.30 \mathrm{~g}$ in Groups I and II, respectively $(p=0.002)$. Mean weight percentile was 36.57 and 2.91 in Groups I and II, respectively $(p<0.000)$. Despite similar gestational age, APGAR score at $10 \mathrm{~min}$. was statistically lower in Group II (9.7 vs. 8.65; $p=0.045$ ), with no differences in the earlier scores. Despite the $p$-value of $<0.05$, we are of the opinion that our sample size was insufficient to suggest the tendency to late respiratory and adaptive distress in hypotrophic neonates.

Cesarean section was performed in 18 and 18 patients from Groups I and II. The analysis revealed differences in the indications for this mode of delivery. In Group I, the main reason was a sharp increase in blood pressure, which was observed in 12 patients during childbirth or pregnancy. In Group II, this clinical situation occurred in 6 patients. Threatening intrauterine asphyxia was the reason for cesarean delivery in 2 patients from Group I and 6 from Group II. All patients received immunosuppressive drugs in two basic schemes. The first regimen consisted of prednisone with cyclosporine A, second of prednisone with tacrolimus. Two patients in Group II used azathioprine as a third drug. All data are shown in Table 1.

Other clinical problems affecting the diagnosis of preeclampsia were analyzed as well. Eclampsia occurred only in 2 patients, both from Group I. Proteinuria, which is defined

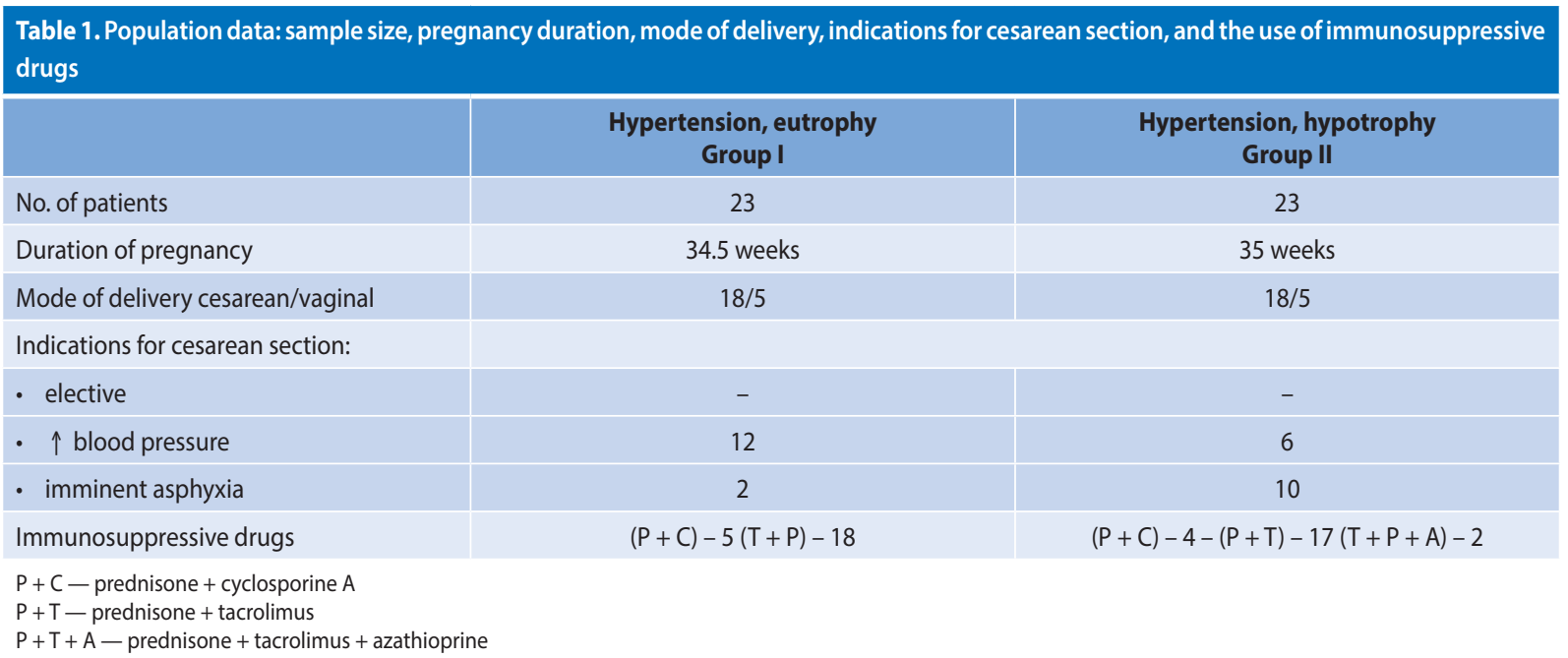




\begin{tabular}{|l|c|c|}
\hline & $\begin{array}{c}\text { Hypertension - eutrophic fetus } \\
\text { Group I }\end{array}$ & $\begin{array}{c}\text { Hypertension + hypotrophy } \\
\text { Group II }\end{array}$ \\
\hline Eclampsia & 2 & 0 \\
\hline Proteinuria before/after 20 weeks & $16 / 22$ & $14 / 20$ \\
\hline Proteinuria $>20$ weeks (de novo) & 6 & 6 \\
\hline Increase in RR $>20$ weeks & 10 & 8 \\
\hline Creatinine $<1.2$ before 20 weeks & 15 & 9 \\
\hline Creatinine $>1.2$ after 20 weeks & 8 (de novo 5) & 8 (de novo 4) \\
\hline Preeclampsia & 21 & 23
\end{tabular}

as the loss of $>300 \mathrm{mg}$ of protein per day, was observed in the first half of pregnancy in 16 and 14 patients from Groups I and II, respectively. It has increased in the second half of pregnancy in 6 patients from Group I and 6 from Group II. The highest recorded loss of protein into urine was $3.8 \mathrm{~g} /$ day, and $9.1 \mathrm{~g} /$ day in Groups I and II, respectively.

Patients from Group II were more prone to urinary tract infections ( 0.43 vs. $0.79 ; p=0.02)$. In 10 patients from Group $\mathrm{I}$, a deterioration of blood pressure control was observed in the second half of pregnancy. It required increased doses of hypotensive drugs or the use of additional medications. A similar situation was observed in 8 patients from Group II.

In the first half of gestation, the concentration of creatinine in blood serum was $<1.2 \mathrm{mg} \%$ in 15 patients from Group I and in 9 from Group II. Higher creatinine concentration was observed in 8 patients from Group I, wherein in 5 the serum creatinine level increased de novo after 20 weeks of pregnancy. Similarly, also 8 patients from Group II presented elevated serum creatinine after 20 weeks of pregnancy, wherein the de novo increase was observed in 4 women.

Based on the abovementioned data, the diagnosis of preeclampsia was made in 21 and 23 patients from Groups I and II, respectively. In Group II, intrauterine hypotrophy was the only cause for the diagnosis in 2 cases, while signs of renal dysfunction were the basis for the diagnosis in the remaining 21 patients. Detailed data are shown in Table 2.

\section{DISCUSSION}

Preeclampsia is one of the most frequently reported complications in pregnancies after kidney transplantation $[4,5]$. This complication is up to 8 times more common in that group of women than in the general population [5]. Due to the fact that the etiology of preeclampsia remains to be fully elucidated, every few years its classification changes, making it difficult to compare patients from the past, especially after 2014.

Furthermore, proper qualification and diagnosis, relevant to the clinical symptoms, present a challenge also in other groups of patients. Similar problems have been reported about patients with chronic kidney disease and systemic lupus erythematosus (SLE). Preeclampsia affects up to $30 \%$ of pregnancies in these women $[6,7]$. Out of these all conditions, clinical diagnosis of preeclampsia is much more difficult because most of these patients had hypertension and proteinuria before pregnancy $[8,9]$. The diagnosis is based on deterioration of hypertension control and increased proteinuria, combined with lowered platelet count, elevated liver enzymes, or intrauterine fetal hypotrophy $[10,11]$. Worsening of hypertension and proteinuria may reflect worsening of the underlying disease or the physiological changes in pregnancy. Differentiation between these symptoms is very important because the course of action in each of these cases is different [10]. Sometimes preeclampsia is the first sign of chronic kidney disease [12].

In our study, no significant differences in pregnancy duration (35 weeks in Group I vs. 34.5 weeks in Group II), time from transplant to delivery, or BMI were found, which is consistent with the findings of other authors [13]. Mode of delivery was similar in both groups, with the majority of cesarean sections, although indications for the procedure were different: mainly a sudden increase in blood pressure during pregnancy or childbirth in Group I and threatening intrauterine fetal asphyxia in Group II.

Chronic immunosuppressive therapy constitutes an important factor, which undoubtedly has an impact on the fetal and neonatal outcome. The most frequently used immunosuppressive drugs in pregnant after kidney transplantation include prednisone, azathioprine, cyclosporine A, and tacrolimus. Prednisone passes through the placenta but owing to its rapid metabolism by placental $11 \beta$-hydroxysteroid dehydrogenase, fetal serum concentration reaches only $10 \%$ of the maternal levels. Azathioprine and 6-methylmercaptopurine (the active metabolite of azathioprine) also pass through the placenta. Azathioprine concentration in the placenta is relatively high, reaching $64-93 \%$ of the concentration in the maternal serum. However, the concentration in fetal blood is much lower, and is $1-5 \%$ of that in the maternal serum [14-16]. Cyclosporine A cross the placenta, reaching about $5 \%$ fetal blood concentration compared to 
the concentration in the maternal serum [14-18]. Systematic literature review published in 2016 by the European League Against Rheumatism shows compatibility with pregnancy and lactation for azathioprine, cyclosporine, tacrolimus and glucocorticosteroids [19].

Notably, out of 48 pregnancies, pregestational hypertension and proteinuria $<20$ weeks of gestation were found in most patients (46:16 in Group I and 14 in Group II). Both of these factors are known risk factors for intrauterine growth retardation [20]. Deteriorated blood pressure control was found in 10 patients from Group I and in 8 from Group II. Proteinuria has markedly increased in the second half of pregnancy in 6 patients from Group I and 6 in Group II. Based on the existing criteria, preeclampsia should be diagnosed in all of these patients. Analyzing other clinical parameters, there was no difference in the increase of serum creatinine levels $>20$ weeks of pregnancy ( $>1.2 \mathrm{mg} \%$ ) ( 8 patients from Group I and 8 from Group II). Taking into account all of the abovementioned parameters, if we strictly abide by the recommendations, preeclampsia should be recognized in the vast majority of patients after kidney transplant - according to our observations in 46 from 48 patients. Noteworthy, none of the preeclampsia markers mentioned above had any effect on the condition of the neonate delivery.

Three factors seem to have a significant impact on the possibility of intrauterine growth retardation and preeclampsia in post-transplant patients. The exact etiopathogenesis of eclampsia is still a topic of much debate, but recently researches have been paying more attention to the role of damage to the vascular endothelium [21], which is especially true about the vulnerable patients with chronic kidney disease. Hypertension and proteinuria are found in the majority of the affected subjects, while immunosuppressive therapy is observed in all patients. In our study, no differences in these factors have been noted. Therefore, intrauterine hypotrophy in these patients proves to be an independent factor, sufficient for the recognition of preeclampsia. Neonatal outcomes have been correlated more directly with the intensity of hypotrophy than with gestational age alone.

\section{CONCLUSIONS}

Current diagnostic criteria for preeclampsia are insufficient in pregnant women after kidney transplantation. General criteria should be applied with special care in women with chronic kidney disease or with systemic lupus erythe- matosus. In those patients, intrauterine growth restriction seems to be a better predictive factor for neonatal morbidity than typical markers of kidney function.

\section{REFERENCES}

1. Regulation of the Minister of Health dated 9 November 2015. On standards for medical procedure by providing medical services in the field of obstetrics and gynecology in the field of perinatal care obstetric-gynecological, exercised over a woman during pregnancy, childbirth, postpartum, in the cases of certain complications and the care of the woman in a situation of poor obstetric; Official Gazette 2015 item. 2007 announced 1.12.2015r.

2. The classification, diagnosis and management of the hypertensive disorders of pregnancy: A revised statement from the ISSHP. Pregnancy Hypertension. Int J Women's Cardiovasc Health. 2014, 4, 97-104.

3. Hypertension in pregnancy. Report of the American College of Obstetricians and Gynecologists' Task Force on Hypertension in Pregnancy. American College of Obstetricians and Gynecologists, the Task Force on Hypertension in Pregnancy. Obstet Gynecol. 2013, 122, 1122.

4. Wyld ML, Clayton PA, Jesudason S, [et al.]. Pregnancy outcomes for kidney transplant recipients. Am J Transplant. 2013, 13, 3173.

5. Deshpande NA, James NT, Kucirka LM, Boyarsky BJ, [et al.]. Pregnancy outcomes in kidney transplant recipients: a systematic review and meta-analysis. Am J Transplant. 2011, 11, 388-404.

6. Kwok LW, Tam LS, ZhuT, [et al.]. Predictors of maternal and fetal outcomes in pregnancies of patients with systemic lupus erythematosus. $\mathrm{Lu}$ pus. 2011, 20, 829-836.

7. Chakravarty EF, Colón I, Langen ES, Nix DA, [et al.]. Factors That Prematurity and predict preeclampsia in pregnancies That are complicated by systemic lupus erythematosus. Am J Obstet Gynecol. 2005, 192, 1897.

8. Bermas B. Pregnancy in women with systemic lupus erythematosus. Literature review current through: UpToDate.com, Apr 2016.

9. August $P$, Vella J. Pregnancy in women with underlying renal disease. Literature Review current through: UpToDate.com, Apr 2016.

10. August P, Sibai BM. Preeclampsia: Clinical features and diagnosis. Literature review current through: UpToDate.com, Apr 2016.

11. Nevis IF, Reitsma A, Dominic A, [et al.]. Pregnancy outcomes in women with chronic kidney disease: a systematic review. Clin J Am Soc Nephrol. 2011, 6, 2587-2598.

12. Ihle BU, Long P, Oats J, Early onset pre-eclampsia: recognition of underlying renal disease. BrMed J (Clin Res Ed). 1987, 294, 79.

13. Vázquez-Rodríguez JG, Ríos-Chavarría AL. Perinatal complications in women with kidney transplant. Nephrology. 2012, 32, 639-646.

14. Winkler M. Cyclosporin as baseline immunosuppression in solid organ transplantation. BioDrugs. 2000, 14, 185-193.

15. Prevot A, Martini S, Guignard JP. In utero exposure to immunosuppressive drugs. Bio Neonate. 2002, 81, 73-81.

16. Jabiry-Zieniewicz Z, Dabrowski FA, Pietrzak B, [et al.]. Pregnancy in the liver transplant recipient. Liver Transpl. 2016. doi: 10.1002/lt.24483.

17. Tendron A, Gouyon JB, Decramer S. In utero exposure to immunosuppressive drugs: experimental and clinical studies. Pediatr Nephrol.2002, 17, 121-130.

18. Fischer T, Neumayer HH, Fischer R, [et al.]. Effect of pregnancy on long-term kidney function in renal transplant recipients treated with cyclosporine and azathioprine with. Am J Transplant. 2005, 5, 2732-2739.

19. Götestam C, Hoeltzenbein M, Tincani A, [et al.]. The EULAR points to Consider for use of antirheumatic drugs before pregnancy, and during pregnancy and lactation. Ann Rheum Dis. doi: 10.1136/annrheumdis-2015-208840.

20. Wikström AK, Gunnarsdottir J, Nelander M, [et al.]. Prehypertension in Pregnancy and Risks of Small for Gestational Age Infant and Stillbirth. Hypertension. 2016, 67, 640-646. doi: 10.1161/HYPERTENSIONAHA.115.06752.

21. Phipps E, Prasanna D, Brima W, [et al.]. Preeclampsia: Updates in Pathogenesis, Definitions, and Guidelines. Clin J Am Soc Nephrol. 2016 Apr 19. regimens: CJN.12081115. [Epub ahead of print]. 